\title{
Zwischen Dynamik und \\ Synchronisation. Herausforderungen \\ und Handlungsoptionen für die \\ strategische hochschulweite digitale \\ Transformation der Hochschullehre
}

\section{Marcel Graf-Schlattmann, Birte Thomsen, Melanie Wilde, Dorothee M. Meister und Gudrun Oevel}

\section{Zusammenfassung}

Im vorliegenden Beitrag verbinden wir drei Aspekte der Digitalisierung der Hochschullehre miteinander und diskutieren sie im Verhältnis zu Strategieempfehlungen und Modellen der Agilität. Zuerst stellen wir dabei die Gelingensbedingungen der strategisch gerahmten Digitalisierung anhand unseres Modells der Kollektiven Veränderung dar. Anschließend widmen wir uns der Projektförmigkeit als dem zentralen Organisationsmuster der digitalen Transformation sowie dem Verhältnis von Dynamik und Synchronisation. Beide Phänomene sind wichtige Eigenschaften der digitalen Transformation, die eine Herausforderung, aber auch eine Chance darstellen.

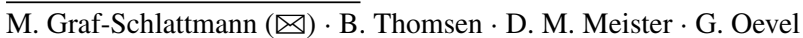
Universtität Paderborn, Paderborn, Deutschland

E-Mail: marcel.graf.schlattmann@uni-paderborn.de

B. Thomsen

E-Mail: birte.thomsen@uni-paderborn.de

D. M. Meister

E-Mail: dorothee.meister@uni-paderborn.de

G. Oevel

E-Mail: gudrun.oevel@uni-paderborn.de

M. Wilde

Universtität Bielefeld, Bielefeld, Deutschland

E-Mail: melanie.wilde@uni-bielefeld.de
} 


\section{Schlüsselwörter}

Erfolgsfaktoren • Kollektive Veränderung • Agilität •

Digitalisierungsprojekte $\bullet$ Change Management $\bullet$ Digitale Transformation $\bullet$ Hochschullehre

\section{$1 \quad$ Einleitung}

Die hochschulweite Digitalisierung der Hochschullehre stellt die Hochschulen vor eine Vielzahl unterschiedlicher Herausforderungen. Diese sind kein neues Phänomen, denn bereits seit Jahren wird theoretisch, empirisch und mit Hilfe von Modellen über die digitale Transformation in der Hochschullehre diskutiert (Euler 2004; Euler und Seufert 2007). Auch das Hochschulforum Digitalisierung (HFD) beschäftigt sich mit dem Thema und entwickelte Modelle und Handlungsempfehlungen (Schmid und Baeßler 2016; Dräger et al. 2017) für den Prozess der Digitalisierung an den Hochschulen. Hinzu kommen noch Hinweise im Zuge von Peer-to-Peer-Beratungen, etwa zu Strategien für die Hochschullehre im digitalen Zeitalter (Schünemann und Budde 2018) oder Digitalstrategie, Struktur und Kultur an Hochschulen (Ladwig 2020).

Im BMBF-geförderten Forschungsprojekt „Qualitätssicherung in der Digitalisierungsstrategie“ (QuaSiD) fragten wir danach, welchen Herausforderungen sich die Hochschulen im Digitalisierungsprozess stellen müssen und welche Gelingensbedingungen für eine Verankerung und nachhaltige Implementierung von Digitalisierungsprojekten zentral sind. Da Projekte die gängige Hochschulpraxis der digitalen Transformation darstellen, sind sie Gegenstand unserer Forschung. Wie dies schon bei ähnlichen Fragestellungen entwickelt wurde, zeigen auch unsere Untersuchungen auf, dass der Erfolg einer hochschulweiten digitalen Transformation von einer Vielzahl von Faktoren abhängig ist und nicht auf einen einzelnen Erfolgsfaktor oder ein Best-Practice-Modell minimiert werden kann (Graf-Schlattmann et al. 2019a; 2020a). Stattdessen zeigen wir Handlungsvariablen und Gelingensbedingungen auf, die als Erfolg versprechender Rahmen dienen und von den jeweiligen Hochschulen und Fächern situationsadäquat bespielt werden.

Über bisherige Erkenntnisse hinaus entwickelten wir auf Basis empirischer Materialien in Anlehnung an die Grounded Theory das Modell der Kollektiven Veränderung als eine solche Gelingensbedingung, die im Folgenden knapp dargestellt wird. 
Der Beitrag befasst sich mit zwei Phänomenen der Kollektiven Veränderung: zum einen mit der Projektförmigkeit als zentralem Organisationsmuster der digitalen Transformation im Hochschulwesen und zum anderen mit dem Verhältnis von Dynamik und Synchronisation, das sich hinsichtlich einer nachhaltigen Verbreitung und Verankerung von Digitalisierungsprojekten als erfolgskritisch darstellt. Unsere Ergebnisse stellen wir abschließend in den aktuellen Diskussionszusammenhang zu Strategieempfehlungen anderer und zur Diskussion um Agilität und Digital Leadership.

\section{Das Modell der Kollektiven Veränderung}

Die Digitalisierung an Hochschulen ist inzwischen fortgeschritten, auch wenn gerade in Bezug auf die Lehre weiterhin erheblicher Bedarf festgestellt wird (Berinfor 2017; Hochschulforum Digitalisierung 2016). Im Folgenden erläutern wir zunächst das Modell der Kollektiven Veränderung und betten es in den Hintergrund aktueller Diskurse ein.

\subsection{Entwicklung und Bestandteile des Modells der Kollektiven Veränderung}

Beim Vorhaben, die Gelingensbedingungen für die Qualitätssicherung in der Digitalisierungsstrategie zu untersuchen, wählten wir eine mehrdimensionale Herangehensweise. Zunächst wurde in Literaturstudien ein theoriegeleitetes Verständnis des Strategie- und Digitalisierungsbegriffs im Hochschulkontext erarbeitet (GrafSchlattmann et al. 2018, 2019a). Im empirischen Zugang wurde ein Sampling von 27 Hochschulen entlang unterschiedlicher Hochschultypen, Reichweiten und Fachkulturen gewählt, weiter wurden verschriftlichte Digitalisierungsstrategien untersucht und aus dem Sampling heraus 15 leitfadengestützte Experteninterviews mit Akteur*innen in unterschiedlichen Funktionen an jeweils 7 Hochschulen und Universitäten in Deutschland durchgeführt und - gemeinsam mit den weiteren Dokumenten - in Anlehnung an die Grounded-Theory-Methodologie (Glaser und Strauss 1971; Strauss 1998; Strübing 2008) aufbereitet und analysiert. Die in mehreren Iterationen herausgearbeiteten Phänomene wurden anschließend mit Peers aus Forschung und Praxis hinsichtlich ihrer Plausibilität validiert (ausführlicher zur Methodik: Graf-Schlattmann et al. 2020a).

Ein zentrales Ergebnis ist die Identifikation von Gelingensbedingungen, die wir in dem Modell der Kollektiven Veränderung zusammengefasst haben. Das 
Modell besteht aus drei Komponenten: die Kollektive Veränderungsbereitschaft, die Veränderungsmöglichkeit und die Veränderungskompetenz (siehe Abb. 1).

Unserer Erkenntnis nach wirken sich jede Komponente und die dazugehörigen Handlungsvariablen der Kollektiven Veränderung auf unterschiedliche Weise auf das Gelingen des digitalen Transformationsprozesses aus, wie an anderer Stelle bereits dargestellt (Graf-Schlattmann et al. 2020a; 2020b). Insofern referenzieren wir das Modell im Folgenden lediglich kurz und stellen die Verbindung her für die weitere Betrachtung von Projektförmigkeit sowie Dynamik und Synchronisation.

Wir betonen die Handlungsvariable von Professionalität und Freiräumen im Rahmen der Kollektiven Veränderungsbereitschaft, da diese eine zentrale Stellschraube für die soziale Akzeptanz und das Commitment des akademischen Personals ist, die durch den erkennbaren Nutzen und die Unterstützungsstrukturen gefördert wird. Des Weiteren ergeben sich hieraus bedeutende Merkmale des

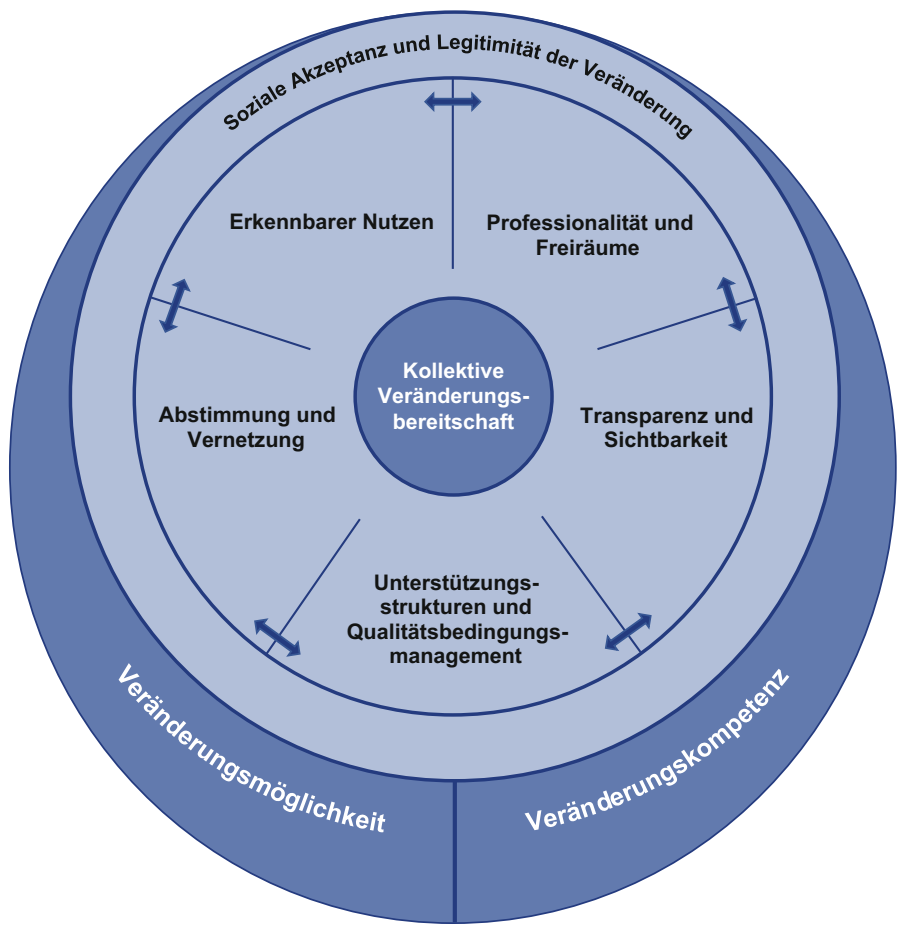

Abb. 1 Das Modell der Kollektiven Veränderung. (Eigene Darstellung) 
Veränderungsprozesses. Das akademische Personal verfügt im Kontext der Professionalität und Freiräume über weitgehende Autonomie im Prozess und in der Wahl der Tools. Durch gewährte Frei- und Experimentierräume entsteht eine Vielzahl von dynamischen Einzelprozessen und sich wiederholenden Schleifen mit unterschiedlichen Geschwindigkeiten, die den Digitalisierungsprozess an Hochschulen kennzeichnen. Hinzu kommt, dass diese Schleifen aufgrund der losen Kopplung als Strukturmerkmal der Hochschulorganisation (Weick 1976) weitgehend unabhängig voneinander erfolgen können. Hieraus ergibt sich ein Synchronisationsbedarf, der die Prozesse hinsichtlich einer gemeinsamen Entwicklungsrichtung eint (Graf-Schlattmann 2018; 2019b).

Auf Basis der Interviews konnten wir für diese Funktion der Synchronisierung die Handlungsvariablen Transparenz und Sichtbarkeit sowie Abstimmung und Vernetzung herausarbeiten. Beide fördern die Akzeptanz, einen aber gleichzeitig auch den Prozess hinsichtlich eines gemeinsamen strategischen Rahmens. Im Hinblick auf Transparenz und Sichtbarkeit werden die Prozesse, aber auch die einzelnen Akteur*innen sichtbar. Dies wird durch die Handlungsvariable von Abstimmung und Vernetzung mit den dazugehörigen Austauschformaten - beispielsweise einem Tag der Lehre oder einem Jour fixe - intensiviert. Auch die typischen gremienbasierten Austausch- und Entscheidungswege der Hochschulen sind hier verortet (Graf-Schlattmann et al. 2020a).

Ergänzend braucht es klare Verantwortlichkeiten - als Teil der Veränderungsmöglichkeit -, die den synchronisierenden Gegenpol zu der Dynamik der Handlungsvariablen Professionalität und Freiräume darstellen. Dabei hat die Hochschulleitung eine besondere Rolle inne. Sie muss den Digitalisierungsprozess anstoßen, gestaltend fördern und eine Gesamtstrategie zur Digitalisierung im Sinne eines strategischen Rahmens (Graf-Schlattmann et al. 2018; 2019b) entwickeln. Es gilt hierbei auch Prioritäten zu setzen und diese in den Entwicklungsplänen der Hochschule zu verankern, um ein gemeinsames Verständnis zu entwickeln und zu dokumentieren.

Die klaren Verantwortlichkeiten erschöpfen sich nicht auf Ebene der Hochschulleitung, sondern müssen auf allen Ebenen der Hochschule greifen. Für einen kontinuierlichen Ablauf ist zudem die Funktion einer Prozessverantwortlichkeit notwendig. Dabei ist die synchronisierende Funktion einer/eines digitalen Netzwerker*in als Bindeglied zwischen den verschiedenen Strängen und iterativen Prozessen hervorzuheben. Dadurch werden die aus der Lehre kommenden iterativen Prozessschleifen in eine gemeinsame Entwicklungsrichtung - den strategischen Rahmen - zusammengeführt.

Für den Prozesserfolg müssen die Handelnden über die relevanten (Veränderungs-) Kompetenzen verfügen. Neben der Handlungskompetenz - der 
durch didaktische und technische Weiterbildungen geförderten Fähigkeit, im Bereich digitaler Lehre zu handeln - braucht es insbesondere eine Entscheidungskompetenz, die auf den unterschiedlichen Ebenen des Prozesses jeweils anders ausgeprägt sein kann. Zum einen geht es darum, die einzelnen Stränge zusammenzuführen und die Synchronisation so zu begleiten, dass Innovationen in Regelstrukturen überführt werden. Hier greift erneut die Funktion der digitalen Netzwerker*innen. Aber auch die Aufgaben der Leitungsebene, den Prozess federführend $\mathrm{zu}$ begleiten, systematisch zu fördern sowie zu verbreitern und die Fähigkeit, einen Überblick über den Prozess, die Ziele und den strategischen Rahmen zu bewahren, ist erfolgskritisch für die nachhaltige Verbreitung und Verankerung der Entwicklung (Graf-Schlattmann et al. 2020b).

\subsection{Einbettung des Modells in aktuelle Diskurse}

Unser empirisch entwickeltes Modell der Kollektiven Veränderung weist Ähnlichkeiten, aber auch Differenzen zu bestehenden Ansätzen auf. In den Handlungsempfehlungen hinsichtlich der Hochschulstrategien für die Lehre im digitalen Zeitalter des HFD (Schünemann und Budde 2018) wie auch in unseren Ergebnissen steht die strategisch ausgerichtete digitale Transformation im Fokus. Dabei ist ein zentraler Erfolgsfaktor in beiden Untersuchungen, dass die Hochschule eine gemeinsame Vision und ein gemeinsames Verständnis der Transformation benötigt, um einen Erfolg versprechenden Transformationsprozess zu durchlaufen. Auch das Strategieverständnis ist ähnlich gelagert, da in beiden Untersuchungen gezeigt wird, dass es einen ganzheitlichen Veränderungsprozess benötigt, der sowohl Top-down als auch Bottom-up strukturiert ist und den Fächern und Einzelpersonen nötige Freiräume einräumt, die Strategie zu füllen. Dies wird in beiden Fällen mit klaren Verantwortlichkeiten und finanziellen sowie personellen Ressourcen gerahmt - in unserem Modell als Teil der Veränderungsmöglichkeit (Graf-Schlattmann et al. 2020b).

Die Funktion einer Prozessverantwortlichkeit ist ebenso Teil der Handlungsempfehlungen des HFD. Die früheren Handlungsempfehlungen fokussierten dabei eher die Strategieentwicklung und setzen hierfür auf Ebene der Hochschulleitung sowie der strategischen Entscheider*innen an, die die Adressat*innen der Handlungsempfehlungen sind (Schünemann und Budde 2018). Unser Modell setzt den Fokus weniger auf die Strategie selber, sondern vielmehr auf den strategisch gerahmten digitalen Veränderungsprozess (Graf-Schlattmann et al. 2019b; 2020b), widmet sich dem Thema also aus der Perspektive des Change Management. Aus diesem Blickwinkel ist die kollektive Bereitschaft zur Veränderung die 
zentrale Herausforderung des Prozesses: der Erfolg im digitalen Transformationsprozess an Hochschulen ist, unseren Ergebnissen nach, maßgeblich von der sozialen Akzeptanz und der Legitimität der Veränderung abhängig. Im Modell der Kollektiven Veränderung wird daher von den Lehrenden und Studierenden her gedacht und die soziale Akzeptanz der Veränderung erhält eine zentrale Bedeutung. Dieser Aspekt wird heute u. a. auch im Format der Peer-to-PeerStrategieberatung des HFD betont und umgesetzt (Rampelt und Wagner 2020). Zudem stellen Projekte und Agilität in unserem Modell wichtige Elemente des Erfolgs im Transformationsprozess dar, die im Weiteren vorgestellt werden.

\section{Projektförmigkeit in der digitalen Transformation}

Ein zentraler Modus, um neue Themen und Innovationen auszuprobieren und zu entwickeln, ist das befristete Projekt, das im Hochschulwesen prägend ist. Projekte dienen oft dazu, neue Themen, Technologien und Innovationen in die Hochschule zu bringen und sind bei vielen Hochschulen von Relevanz.

„Ich glaube, ein Projekt ist einfach ein gutes Instrument, einen Umbauprozess zu begleiten oder umzusetzen [...] ich glaube, dass, wenn man jetzt das nicht als Projekt macht, mit irgendwelchen Enden, dann würde das zu einer unglaublichen Unruhe führen. Wenn permanent alles umgebaut wird. Deshalb ist das schön, ein Projekt hat ein Ende“ (Interviewpartner*in, Universität 1).

Der Vorteil von Projekten für den Digitalisierungs- und Innovationsprozess liegt in der organisationalen Anbaustruktur. Projekte sind meist nicht in die Organisation und das Alltagsgeschäft integriert, sondern an die bestehenden Strukturen angedockt. Dadurch bleiben sie personell und finanziell weitgehend losgelöst vom Alltagsgeschäft und schaffen so die Möglichkeit, Veränderungen irritationsfrei durchzuführen und Neues auszuprobieren. Durch die vergleichsweise freie Experimentierlogik steigt potenziell die intrinsische Motivation und es entsteht ein Möglichkeitsraum im Sinne geeigneter Finanzierung und geförderter Veränderungsmöglichkeit. Als Herausforderung bleibt die Überführung in den Hochschulalltag - strukturell und finanziell. Unsere Studie zeigt, dass es einen erheblichen Unterschied gibt, wie intern und extern geförderte Projekte auf die Hochschulstrategie wirken. Die externen Projektmittel der verschiedenen Träger bieten eine gute Anschubfinanzierung, schaffen jedoch aufgrund der Befristung keine langfristigen und nachhaltigen Strukturen. 
„Da hatten wir jetzt das Glück, dass wir im Qualitätspakt Lehre zum Zuge gekommen sind [...]. Da wird es natürlich die Herausforderung geben, wenn der Qualitätspakt Lehre nicht verlängert wird oder wir nicht zum Zuge kommen sollten, wie wir dann in den Zwanzigerjahren das finanziert kriegen“ (Interviewpartner*in, Hochschule 6).

Neben den klassischen extern finanzierten Drittmittelprojekten identifizierten wir in unserer Studie als zweiten Typus die internen Projekte. Alle Interviewpartner*innen sprachen von eigenen hochschulinternen Projekttöpfen, die in unterschiedlicher Intensität zur Förderung digitaler Anwendungen in der Lehre eingesetzt werden.

„Wir haben den und den Topf; und es dürfen sich Kolleginnen und Kollegen bewerben. Und es sollte möglichst unbürokratisch von statten gehen. Mit der [...] einzigen Auflage, dass, wenn dieses Projekt durchgeführt wird, es veröffentlicht wird. Und zwar an der Hochschule. Und grundsätzlich als Best Practice dienen muss. Dafür müssen sich die Kollegen bereit erklären. Also sie dürfen sich da nicht in ihr Kämmerlein zurückziehen, die 10.000 verausgaben und sagen: ,War alles Scheiße; will ich nicht'. Sondern DAS müssen sie transparent machen“(Interviewpartner*in, Hochschule 2).

Die Hochschule stellt dabei Mittel für kleine Innovationen zur Verfügung, auf die sich meist Lehrende, selten Studierende bewerben können. Diese Art von Projekten war an allen Hochschulen zu beobachten, der konkrete Nutzen schien jedoch nicht überall gleichermaßen erkannt worden zu sein.

„Ein Projekt wird eingeworben durch ein Institut oder durch einen Verbund von Instituten. Und das bedeutet nicht automatisch, dass das zum Nutzen der Hochschule ist. Wenn ich jetzt für meinen Bereich beispielsweise Forschungsgelder einwerbe, dann schaue ich, dass ich mein Personal damit finanziere. Das tue ich nicht, um der [Universität 2] irgendwas Gutes tun zu wollen. Und deshalb glaube ich, sind diese [Universität 2] internen Projektmittel viel, viel wichtiger für die Umsetzung der Digitalisierungsstrategie als diese Außenfinanzierung. Die Außenfinanzierung ist notwendig, die ist auch wichtig und sie bringt oftmals viel mehr an Geldmitteln. Aber das ist ein anderes Geschäft" (Interviewpartner*in, Universität 2).

Zusätzlich zu den befristeten Ressourcen kann zudem eine eher schwache Einbindung des akademischen Personals in die Organisationsentwicklung der gesamten Hochschule konstatiert werden, was eine strukturelle Herausforderung für die Nachhaltigkeit von extern drittmittelfinanzierten Projekten darstellt (Stichweh 2005; Kleimann 2016; Watolla 2019). Drittmittelfinanzierte Projekte werden zumeist aus einem Eigeninteresse des akademischen Personals beantragt, ohne dass ein direkter Nutzen für die Hochschule angestrebt wird, was die nachhaltige Verbreitung und Verankerung erschwert. Extern finanzierte Projekte bringen 
damit die Digitalisierung durch Innovationen voran und schaffen meist sogenannte Leuchttürme. Die internen Projekte wiederum schaffen selten derartige Innovationen, stattdessen können kleinteiligere Erkenntnisse Themen in die Breite der Hochschule tragen und im Sinne von Experimenten die Entwicklung erlebbar machen. Interne Projektmittel bieten so eine Möglichkeit zum gemeinsamen Handeln innerhalb des strategischen Rahmens.

\begin{abstract}
„Also wenn Sie jetzt heute Morgen beispielsweise aufgewacht wären und Sie haben sich überlegt, mit welchen Verfahren Sie Ihre Lehre verbessern. Dann machen Sie dazu einen kleinen Antrag, nicht mehr als fünf Seiten Text. [...] Und wenn Sie jetzt nach der Auffassung der Kommission einen so interessanten Antrag gestellt haben, [...] dann haben Sie wirkliches Wagniskapital gekriegt. [...] Also wenn Sie so ein bestimmtes Format entwickelt haben, dann wird das auch mit Ihrem Namen identifiziert und dann werden auch bestimmte Formate, ja, mit Namen verbunden. [...] dann kontaktieren Sie bitte einen Kollegen X oder eine Frau Kollegin Y. Das heißt, Sie kriegen da auch eine Wertschätzung für, Sie kriegen dafür eine entsprechende Anerkennung“ (Interviewpartner*in, Universität 2).
\end{abstract}

Interne Projekte haben in mehrerlei Hinsicht Auswirkungen auf die nachhaltige Implementierung und Verankerung der Projektergebnisse und -ansätze. Allen voran wird ein niedrigschwelliger Einstieg ins Thema ermöglicht. Durch systematische Ergebnissicherung beispielsweise mit Hilfe von Austauschformaten oder Datenbanken mit den jeweiligen Projekten können die Entwicklungen zusammengeführt und eine verbesserte Sichtbarkeit der Entwicklungen geschaffen werden.

Internen Projekttöpfen kommt eine Unterstützungsfunktion zu, die einerseits geeignet ist, die dazugehörigen Handlungsvariablen - insbesondere die Frei- und Experimentierräume und den erkennbaren Nutzen - zu fördern, und andererseits die am konkreten Bedürfnis der Lehrenden orientierte und akzeptierte Lösungen schaffen kann. Dies weist auf agile Strukturen im Hochschulalltag hin, die auf Innovationen und eine stete Anpassung und Weiterentwicklung bauen und im nächsten Kapitel behandelt werden.

Bedeutende Facetten in der Einbindung interner Projekte in die Digitalisierungsstrategie sind die ausdifferenzierte Vernetzungsfunktion sowie die klaren Verantwortlichkeiten. Durch die Grenzstellenfunktion werden die Ansprüche und Herangehensweisen verschiedener Akteur*innen geeint, die vielen kleinteiligen und dynamischen Einzelprozesse werden miteinander verbunden und in eine gemeinsame Richtung gemäß dem strategischen Rahmen der Hochschule gebracht und können so synchronisiert werden. Die Rolle der/des digitalen Netzwerker*in kann aber auch eine systematische Verbindung der beiden Projekttypen fördern, 
da so die Kopplung der Innovationen aus den Drittmittelprojekten mit denen der Hochschulen intensiviert und der Transfer von Projektergebnissen unterstützt werden kann. Die Prozesse können zwar nicht komplett gesteuert werden, dennoch ist eine Einflussnahme durch Gestaltung förderlicher Bedingungen möglich. Dabei erscheint ein austariertes Gleichgewicht zwischen Dynamik im Sinne von Innovation und Synchronisation wichtig.

\section{$4 \quad$ Dynamik und Synchronisation im Innovationsprozess}

Der Erfolg dieser ausbalancierten Herangehensweise ist von zweierlei Bedingungen bestimmt. Zum einen braucht es eine veränderungs- und innovationsfördernde Kultur und zum anderen eine intensive Vernetzung, um die verschiedenen iterativen Schleifen hinsichtlich eines gemeinsamen strategischen Rahmens zu einen. Die Vernetzung und gleichzeitig stetige kollektive Bewertung von Projekten sind eine notwendige Voraussetzung für die nachhaltige Verbreiterung von Innovation durch Projekte (Oevel et al. 2018) und setzen wiederum die Funktion der Prozessverantwortlichkeit voraus.

Unsere empirischen Ergebnisse sowie die Feedbackschleife mit Peers ${ }^{1}$ zeigen einen interessanten Zusammenhang zur Diskussion um Agilität und Digital Leadership auf. Hochschulen gelten gemeinhin als hochgradig behäbig und veränderungsresistent (beispielsweise Stock 2004; Schimank 2005; Camilleri et al. 2019). Zwar wurde bereits im Zuge des New-Public-Managements versucht diese strukturelle Behäbigkeit abzumildern und die Organisation durch verstärkte interne Hierarchie und Schwächung der akademischen Selbstverwaltung flexibler zu gestalten (Meier 2009; Hüther 2010; Hüther und Krücken 2016). Doch auch heute noch wird argumentiert, dass Hochschulen flexibler und agiler werden müssten (Rahrt 2019; Seidl und Vonhof 2017; Hanft et al. 2017).

Demgegenüber werden jedoch die Arbeitsweisen und -prozesse innerhalb der Hochschulen als hochgradig agil beschrieben (Baecker 2017). Auch unsere Forschungsergebnisse weisen in diese Richtung. Petry (2019) fasst die agilen Mindsets, die allen agilen Organisationsformen - beispielsweise Holacracy, agile Matrixorganisation oder auch agile Projektorganisation durch Design-Thinking, Scrum oder Lean-Start-up - als Denkweise und Handlungslogik zugrunde liegen, in verschiedenen Punkten zusammen. Dabei geht es in allen Ansätzen um

\footnotetext{
${ }^{1}$ Hier danken wir insbesondere Prof. Dr. Kerstin Mayrberger von der Universität Hamburg.
} 
eine funktionierende und von den Kund*innen akzeptierte Lösung, die am tatsächlichen Bedürfnis ausgerichtet ist und in selbstständigen und crossfunktionalen Teams durch iteratives Testen und Lernen entsteht (ebenda, S. 48 ff.).

Sofern man die Lehrenden - bzw. auch die Studierenden - als Akteur*innen, im Sinne von Prosumer*innen oder Nutzer*innen bzw. Anwender*innen, versteht, werden die Parallelen deutlich. Im Zentrum des Erfolgs steht das Bedürfnis bzw. Interesse der Lehrenden, das die konkreten Schritte beeinflusst. Ebenso wie bei einer agilen Produktentwicklung dürfen die Aktivitäten und Angebote nicht am Interesse der Anwender*innen vorbeigehen. Hinsichtlich einer digitalisierten Lehre bedeutet das, dass sowohl die Strategie als Rahmen (Graf-Schlattmann et al. 2018; 2019b) als auch die konkret eingesetzten Tools und Anwendungen gemeinsam mit den Lehrenden und den Studierenden im Zuge der gewährten Frei- und Experimentierräume entwickelt oder gewählt werden. Dies ist wichtig, da das Ziel der Entwicklung die Akzeptanz der Anwender*innen ist - bei Petry (2019) die funktionierende und von den Kund*innen akzeptierte Lösung, im vorliegenden Fall die soziale Akzeptanz und Legitimität der Veränderung als zentraler Bestandteil der Kollektiven Veränderungsbereitschaft. Dies findet sich bereits bei Euler (2004), der den Wandel in den Köpfen der Menschen als zentrales Moment bei der nachhaltigen Implementierung von Innovationen in Regelstrukturen versteht.

In unseren Ergebnissen findet sich dieser Aspekt in den Frei- und Experimentierräumen, die konzeptionell ebenso in der Vorgehenslogik des agilen Mindsets liegen. Durch Experimente und eine Akzeptanz von Fehlern können Lösungen und Anwendungen entwickelt werden, die an den Bedürfnissen und Interessen der Lehrenden und Studierenden orientiert sind. Dieser Gedanke liegt auch der förderlichen Kultur zugrunde, die es neben den gewährten Freiräumen ermöglicht, Fehler zu machen, und vermeintliche Fehlentwicklungen, die zum Entwicklungsprozess dazugehören, toleriert. Eine andere Facette der Kultur ist die bestmögliche Unterstützung der Beteiligten bei ihren Anliegen. Dies findet sich in der zentralen Bedeutung der Unterstützungsstrukturen bzw. des Qualitätsbedingungsmanagements (Pasternack et al. 2018), die in allen drei Komponenten der Kollektiven Veränderung wirken und als selbstständige, crossfunktionale Teams im Sinne des agilen Mindsets funktionieren können. Auch Euler (2004) sowie Euler und Seufert (2007) führen die Kooperationen mit anderen Teams als wichtigen Mechanismus an. All dies führt zu einem gestärkten Commitment der Beteiligten und einer Kultur des Vertrauens innerhalb der Hochschule.

Diese Erkenntnisse finden sich auch in den ergänzenden Führungscharakteristika eines agilen Mindsets - bestehend aus Vernetzung, Offenheit, Partizipation und Agilität und dem Vertrauen in das Wollen und Können der Mitarbeiter*innen 
- sowie im Führungshandeln an Hochschulen mit einem erfolgreichen strategisch gerahmten Digitalisierungsprozess.

Partizipation bedeutet bei Petry (2019) das gemeinsame Entwickeln von Ideen, Konzepten und Prototypen. Dafür muss die Führungskraft die kollektive Intelligenz nutzen und fördern, also die Expertisen der Anwender*innen - der Lehrenden und Studierenden - einholen und einen Rahmen schaffen, in dem sie sich entfalten können (ebenda, S. 49 f.). Dabei wird auch empfohlen, die Selbstorganisation und -steuerung von Expert*innenteams und Communitys zu nutzen und keine manageriale Fremdsteuerung vorzunehmen. Im Hochschulwesen sind die Möglichkeiten zur Steuerung von Forschung und Lehre zusätzlich durch dessen Autonomie erschwert (beispielsweise Kleimann 2016; Pasternack et al. 2018). Der partizipative Gedankengang findet sich auch in dem Baustein von Zielen und Strategien erfolgreicher Unternehmensführung wieder (Petry 2019, S. 74 ff.). Dabei sollten grobe, richtunggebende Antworten auf die Fragen, was sich verändern und wohin die Veränderung führen soll, und Digitalisierungsziele in Partizipation mit allen Stakeholdern entwickelt werden. Dies entspricht in der Konzeption auch unserem Strategieverständnis (Graf-Schlattmann et al. 2018; 2019b) und hebt die Bedeutung der Prozessbeteiligten zusätzlich hervor.

Eine weitere Facette der Führungscharakteristika ist die Offenheit, die sich in unserem Modell darin zeigt, dass in der Handlungsvariablen von Transparenz und Sichtbarkeit ein offenes Feedback möglich ist und auch offen Kritik angenommen wird (Petry 2019, S. 51). Des Weiteren ist hier auch die Fehlerkultur zu verorten. Neben der Offenheit der Kultur wird auch die Sichtbarkeit der Prozesse, die Vernetzung der Akteur*innen sowie die soziale Akzeptanz gefördert. Die Vernetzung hat die bereits diskutierte Funktion, die iterativen Schleifen des Prozesses hinsichtlich einer gemeinsamen Zielrichtung zusammenzuführen. Denn die hier beschriebenen Prozesse wirken sich aufgrund der losen Kopplung als Strukturmerkmal des Hochschulwesens (Weick 1976) nicht auf umliegende Entwicklungen und Disziplinen aus. Dadurch entsteht das dichotome Bild von innovativen Prozessen im Kleinen bei gleichzeitiger Trägheit auf organisationaler Ebene (Graf-Schlattmann i. E.).

Im Bereich agiler Prozesse wird bzgl. des festgestellten Spannungsverhältnisses zwischen Dynamik und Synchronisation von der Ambidextrie oder beidhändigem Führen gesprochen (March 1991; Petry 2019). Dieser Mechanismus, zweierlei Richtungen zusammenzuführen, findet sich in unserem Material an unterschiedlichen Stellen. So ist zum einen das Zweistromprinzip oder auch der Dualismus von Zentralität und Dezentralität zu nennen, zum anderen lässt sich der Gedanke auch in den technischen Infrastrukturen finden, die sowohl basale Grunddienste als auch individuelle Lösungen und Tools abbilden. Auch in den 
Ausprägungen und Verhältnissen zwischen den verschiedenen Handlungsvariablen sollte eine solche Beidhändigkeit vorherrschen. Da die einzelnen Variablen sich wechselseitig bedingen und beeinflussen, kann ein Überstrapazieren der einen Handlungsvariablen sich negativ auf die andere auswirken. Deshalb sollte, als weiterer Erfolgsfaktor, ein kontinuierlicher Veränderungsprozess verankert werden, der die Verbreitung und Verankerung von Innovationen und Projekten einerseits längerfristig und mehrzyklisch angeht und sich andererseits an die sich stetig ändernden - technischen, finanziellen oder personellen - Bedingungen anpassen kann (Euler 2004, S. 565 f.). Die digitale Transformation ist also nicht in Phasen des „Unfreeze-Move-Refreeze“ (Themengruppe 2015) zu unterteilen, sondern sollte als „chronocally unfrozen“ (Weick 1977) verstanden und angegangen werden.

\section{$5 \quad$ Fazit}

Für den Erfolg einer hochschulweiten Digitalisierung erscheint das Schaffen kollektiver Veränderungsbereitschaft durch das wechselseitige Zusammenspiel von sechs Handlungsvariablen von zentraler Bedeutung zu sein. Die kollektive Veränderungsbereitschaft wird von der Veränderungsmöglichkeit und -kompetenz hinsichtlich der Umsetzbarkeit gerahmt. Insgesamt zeigen unsere Analysen, dass eine übergeordnete und erfolgskritische Anforderung im digitalen Veränderungsprozess die Synchronisation verschiedener Dynamiken im Sinne eines situativen Gleichgewichts zwischen Dynamik und Synchronisation ist, weshalb der Erfolg nicht auf einen einzelnen Faktor oder ein Best-Practice-Modell minimiert werden kann (Graf-Schlattmann et al. 2019a; 2020a).

Dabei ist es eine zentrale Herausforderung, die grundlegenden Innovationsdynamiken der Digitalisierung und die - häufig befristeten oder projektbasierten - Beschäftigungsstrukturen an den Hochschulen sowie die gremienbasierten Entscheidungswege und demokratisch gewählten Amtsperioden in der akademischen Selbstverwaltung zusammenzuführen. Unser empirisches Material zeigt darüber hinaus, dass sich eine Vielzahl kleinteiliger iterativer Schleifen entwickeln, die ebenso zu einem erhöhten Synchronisationsbedarf führen. Die kleinteiligen Schleifen und projektbasierten Prozesse fördern Innovationen. Diese müssen jedoch nachhaltig implementiert und in verlässliche und trotzdem agile Organisationsstrukturen überführt werden. Dies hat auch zur Folge, dass der Wandel nicht radikal, sondern aufeinander aufbauend und evolutionär erfolgt (Graf-Schlattmann 2018; 2019a). Unsere im Modell Kollektiver Veränderung 
zusammengefassten Ergebnisse zeigen in seinen Mechanismen und Gelingensbedingungen deutliche Parallelen zu sogenannten agilen Ansätzen. Wichtig erscheint es uns für einen erfolgreichen Veränderungsprozess, bei Veränderungskompetenz Schwerpunkte nicht nur auf den Umgang mit Veränderung, sondern auch auf Entscheidungskompetenz in unsicherer Entwicklung zu legen.

Ein weiteres Ergebnis unserer Untersuchungen ist die Unterscheidung von befristeten Projekten hinsichtlich ihrer Implementierung von erfolgreichen Strategien. Projekte haben relevante Funktionen im Innovationsprozess und fördern die Entwicklung der Digitalisierung auf unterschiedliche Weise. So kann mithilfe von Projekten weitgehend irritationsfrei und losgelöst vom Alltagsgeschäft (personell, finanziell) gearbeitet werden und es entstehen Experimentierräume für Innovationen. Gleichzeitig sind sie jedoch bezüglich der Nachhaltigkeit mit Herausforderungen verbunden. Die extern finanzierten Projekte wenden sich dabei stärker einer forschungsorientierten Gesamtentwicklung im Zuge der Digitalisierung zu und sind abgelöster von der breitenorientierten Entwicklung. Anders als die drittmittelfinanzierten Projekte dienen interne Projekttöpfe oft nicht der Tiefe der Innovation, sondern insbesondere der nachhaltigen Implementierung und Verstetigung der digitalen Transformation in der eigenen Hochschule. Also scheinen interne Projektformen eine geeignete Lösung für die Etablierung nachhaltiger Entwicklungen zu sein, die im situativen Gleichgewicht von Dynamik und Synchronisation die bestehenden Fördermaßnahmen und Hochschulstrategien sinnvoll ergänzen.

Während der Coronapandemie sehen wir aktuell erfreulicherweise eine sehr hohe Dynamik an allen Hochschulen. Es wird aus unserer Sicht darauf ankommen, diese Dynamik nachhaltig zu verankern und ein gemeinsames neues Bewusstsein für die digitale Lehre an jeder Hochschule zu synchronisieren.

\section{Literatur}

Baecker, D. (2017). Agilität in der Hochschule. Die Hochschule Journal für Wissenschaft und Bildung, 26(1), 19-28.

Berinfor. (2017). Befragung. Die digitale Zukunft der Hochschule - Wie sieht sie aus und wie lässt sie sich gestalten? https://www.berinfor.ch/assets/docs/befragung/2017-Bericht-Bef ragung-Berinfor-Die-digitale-Zukunft-der-Hochschule.pdf. Zugegriffen: 28. Apr. 2020.

Camilleri, A. F., Werner, T., Hoffknecht, A., \& Sorge, A. (2019). Blockchain in der Hochschulbildung. Essen: Edition Stifterverband. 
Dräger, J., Friedrich, J.-D., Mordhorst, L., Müller, U., \& Röwert, R. (2017). Hochschulen brauchen Strategien für das digitale Zeitalter. In Rat für Forschung und Technologieentwicklung (Hrsg.), Zukunft und Aufgaben der Hochschulen. (S. 263-278) Wien: LIT.

Euler, D. (2004). Gestaltung der Implementierung von E-learning-Innovationen: Förderung der Innovationsbereitschaft von Lehrenden und Lernenden als zentrale Akteure der Implementierung. In D. Euler \& S. Seufert (Hrsg.), E-learning in Hochschulen und Bildungszentren (S. 561-584). München: Oldenbourg.

Euler, D., \& Seufert, S. (2007). Change Management in der Hochschullehre: Die nachhaltige Implementierung von e-Learning-Innovationen. https://www.researchgate.net/public ation/255616920_Change_Management_in_der_Hochschullehre_Die_nachhaltige_Imp lementierung_von_e-Learning-Innovationen/download. Zugegriffen: 28.04.2020.

Glaser, B. G., \& Strauss, A. L. (1971). The discovery of grounded theory: Strategies for qualitative research. Chicago: Aldine-Atherton.

Graf-Schlattmann, M. (i. E.). Hochschulorganisation \& Digitalisierung. Die Auswirkungen organisationaler Funktionslogiken auf den Digitalisierungsprozess an Universitäten. (Dissertation).

Graf-Schlattmann, M., Meister, D. M., Oevel, G., \& Wilde, M. (2018). Hochschulstrategie als Prozess. Zum allgemeinen und hochschulspezifischen Begriff der Strategie, QuaSiD (Arbeitspapier, 1). https://doi.org/10.5281/zenodo.1293797.

Graf-Schlattmann, M., Wilde, M., Meister, D. M., \& Oevel, G. (2019a). Digitaler Wandel als strategischer Transformationsprozess - Zum allgemeinen und hochschulspezifischen Verständnis der Digitalisierung. https://doi.org/10.5281/zenodo.2589943.

Graf-Schlattmann, M., Meister, D. M., Oevel, G., \& Wilde, M. (2019b). Digitalisierungsstrategien auf dem Prüfstand - eine empirische Untersuchung auf Basis der GroundedTheory-Methodologie an deutschen Hochschulen. In J. Hafer, M. Mauch, \& M. Schumann (Hrsg.), Teilhabe in der digitalen Bildungswelt (S. 14-26). Münster, New York: Waxmann.

Graf-Schlattmann, M., Meister, D. M., Oevel, G., \& Wilde, M. (2020a). Kollektive Veränderungsbereitschaft als zentraler Erfolgsfaktor von Digitalisierungsprozessen an Hochschulen. Zeitschrift für Hochschulentwicklung, 15(1), 19-39.

Graf-Schlattmann, M., Meister D. M., Oevel G., \& Wilde M. (2020b). Gelingensbedingungen für die strategisch gerahmte Digitalisierung der Hochschullehre. Tagungsband der 15. Jahrestagung der GFHF 2020.

Hanft, A., Maschwitz, A., \& Stöter, J. (2017). Agiles Projektmanagement an Hochschulen - get the things done. Synergie Fachmagazin für Digitalisierung in der Lehre, 3, 8-16.

Hochschulforum Digitalisierung (2016). The Digital Turn - Hochschulbildung im digitalen Zeitalter (Arbeitspapier, 27). Berlin: Hochschulforum Digitalisierung. https://doi.org/10. 5281/zenodo.4282305.

Hüther, O., \& Krücken, G. (2016). Hochschulen. Fragestellungen, Ergebnisse und Perspektiven der sozialwissenschaftichen Hochschulforschung. Wiesbaden: Springer VS.

Hüther, O. (2010). Von der Kollegialität zur Hierarchie? Analyse des New Managerialism in den Landeshochschulgesetzen. Wiesbaden: Springer VS.

Ladwig, T. (2020). Konsequenzen der Digitalisierung für Rechenzentren. Strategie-StrukturKultur am Beispiel der HOOU an der TUHH. Vortrag auf der ZKI Frühjahrstagung 2020. https://www.zki2020.de/custom/media/ZKI_2020/Referenten/ZKI_2020_Donners tag_TinaLadwig.pdf. Zugegriffen: 28. Apr. 2020. 
March, J. G. (1991). Exploration and exploitation in organizational learning. Organization Science, 1, 71-87.

Meier, F. (2009). Die Universität als Akteur: zum institutionellen Wandel der Hochschulorganisation. Wiesbaden: Springer VS.

Orton, J. D., \& Weick, K. E. (1990). Loosely coupled systems: a reconceptualization. Academy of Management Review, 15(2), 203-223. https://doi.org/10.5465/amr.1990.4308154.

Kleimann, B. (2016). Universitätsorganisation und präsidiale Leitung. Führungspraktiken in einer multiplen Hybridorganisation. Wiesbaden: Springer Fachmedien.

Oevel, G., Budde J., \& Wilde M. (2018). Lehr-/Lerninnovationen nachhaltig gestalten. Ein Leitfaden. Paderborn: Universität Paderborn. https://doi.org/10.5281/zenodo.1291408.

Pasternack, P., Schneider, S., Trautwein, P., \& Zierold, S. (2018). Die verwaltete Hochschulwelt Reformen, Organisation, Digitalisierung und das wissenschaftliche Personal. Berlin: BWV - Berliner Wissenschafts-Verlag.

Petry, T. (2019). Digital Leadership - Unternehmens- und Personalführung im digitalen Zeitalter. In T. Petry (Hrsg.), Digital Leadership Erfolgreiches Führen in Zeiten der Digital Economy, (2. Aufl., S. 23-126). Freiburg: Haufe-Lexware.

Rahrt, R. (2019). Digitale Hochschulen brauchen agile Strukturen. HFD-Blog. https://hoc hschulforumdigitalisierung.de/de/blog/digitale-hochschulen-brauchen-agile-strukturen. Zugegriffen: 28.04.2020.

Rampelt, F., \& Wagner, B. (2020). Digitalisierung in Studium und Lehre als strategische Chance für Hochschulen. In R. Stang \& A. Becker (Hrsg.), Zukunft Lernwelt Hochschule. Perspektiven und Optionen für eine Neuausrichtung (S. 105-120). Berlin, Boston: De Gruyter Saur.

Schimank, U. (2005). Die akademische Profession und die Universitäten. In T. Kaletzki \& V. Tacke (Hrsg.), Organisation und Profession (S. 143-164). Wiesbaden: Springer VS.

Schmid, U., \& Baeßler, B. (2016). Strategieoptionen für Hochschulen im digitalen Zeitalter (Arbeitspapier, 29). Berlin: Hochschulforum Digitalisierung. https://doi.org/10.5281/zen odo.4282305.

Schünemann, I., \& Budde, J. (2018). Hochschulstrategien für die Lehre im digitalen Zeitalter: Keine Strategie wie jede andere! (Arbeitspapier, 38). Berlin: Hochschulforum Digitalisierung. https://doi.org/10.5281/zenodo.2592258.

Seidl, T., \& Vonhof, C. (2017). Agile Prinzipien - was kann die Studiengangsentwicklung davon lernen? . Synergie Fachmagazin für Digitalisierung in der Lehre, 3, 28-32.

Stichweh, R. (2005). Neue Steuerungsformen der Universität und die akademische Selbstverwaltung. In U. Sieg \& D. Korsch (Hrsg.), Die Idee der Universität heute (S. 123-134). München: K. G. Saur Verlag.

Stock, M. (2004). Steuerung als Fiktion. Anmerkungen zur Implementierung der neuen Steuerungskonzepte an Hochschulen aus organisationssoziologischer Sicht. Die Hochschule. Journal für Wissenschaft und Bildung, 1(13), 30-48.

Strauss, A. L. (1998). Grundlagen qualitativer Sozialforschung. Datenanalyse und Theoriebildung in der empirischen Sozialforschung. München: Fink.

Strübing, J. (2008). Grounded Theory. Zur sozialtheoretischen und epistemologischen Fundierung des Verfahrens der empirisch begründeten Theoriebildung. (2. überarbeitete und (erweiterte). Wiesbaden: Springer VS.

Themengruppe Change Management \& Organisationsentwicklung (2015). Die Verankerung von digitalen Bildungsformaten in deutschen Hochschulen - Ein Großprojekt wie jedes 
andere? (Arbeitspapier, 11). Berlin: Hochschulforum Digitalisierung. https://doi.org/10. 5281/zenodo.4282269.

Watolla, A. (2019). Strategische Weiterentwicklung von Studium und Lehre im digitalen Zeitalter: Handlungsfelder und Herausforderungen. Diskussionspapier, 6. Berlin: Hochschulforum Digitalisierung. https://doi.org/10.5281/zenodo.3484719.

Weick, K. E. (1976). Educational organizations as loosely coupled systems. Administrative Science Quarterly, 1(21), 1-19.

Weick, K. E. (1977). Organization design: organizations as self-designing systems. Organizational Dynamics, 6(2), 31-46.

Open Access Dieses Kapitel wird unter der Creative Commons Namensnennung 4.0 International Lizenz (http://creativecommons.org/licenses/by/4.0/deed.de) veröffentlicht, welche die Nutzung, Vervielfältigung, Bearbeitung, Verbreitung und Wiedergabe in jeglichem Medium und Format erlaubt, sofern Sie den/die ursprünglichen Autor(en) und die Quelle ordnungsgemäß nennen, einen Link zur Creative Commons Lizenz beifügen und angeben, ob Änderungen vorgenommen wurden.

Die in diesem Kapitel enthaltenen Bilder und sonstiges Drittmaterial unterliegen ebenfalls der genannten Creative Commons Lizenz, sofern sich aus der Abbildungslegende nichts anderes ergibt. Sofern das betreffende Material nicht unter der genannten Creative Commons Lizenz steht und die betreffende Handlung nicht nach gesetzlichen Vorschriften erlaubt ist, ist für die oben aufgeführten Weiterverwendungen des Materials die Einwilligung des jeweiligen Rechteinhabers einzuholen. 